\title{
Crossfield response of industrial magnetic sensors
}

\author{
Pavel Ripka, Jan Vyhnánek, Andrey Chirstov \\ Faculty of Electrical Engineering, Czech Technical University \\ Technicka 2, 16627 Praha 6, Czech Republic, ripka@fel.cvut.cz \\ Phone +420 736760601
}

\begin{abstract}
Magnetic sensors used for non-destructive testing, metal detection and other applications are subjected to large perpendicular field. In this paper we show that the existing models describing the response of anisotropic magnetoresistors (AMR) cannot be used for fields larger than the critical value, which is $350 \mu \mathrm{T}$ for the Honeywell HMC1001. This critical value is one order of magnitude lower than the anisotropy field and it is decreasing with increased value of the measured field. From our findings it is clear that AMR sensors cannot be safely used in applications in which fields above $250 \mu \mathrm{T}$ can appear. Neither flipping, nor feedback compensation can compensate for this error. We show that this behaviour is caused by the fact that the single-domain state of the AMR is broken at the mentined critical field. Compared to that, fluxgate sensors including microfluxgates are by principle immune against the crossfield. Unlike in AMR, crossfield sensitivity in fluxgate sensors is second-order effect, which can be kept under control by proper design. We show that even crossfield of $10 \mathrm{mT}$ does not cause significant degradation of the sensor precision.
\end{abstract}

Keywords: Magnetic sensors, crossfield sensitivity, 


\section{Introduction}

Non-linear response to the magnetic field perpendicular to the sensing direction is unwanted property of all magnetic sensors which contain ferromagnetic material [1]. This so called crossfield response can cause serious error of compass, gradiometer or current sensor.

In this paper we discuss crossfield resistance of AMR sensors and integrated fluxgate in the wider field range. Electromagnetic inspection methods often use strong AC or DC excitation field and very small field deviation caused by the defect is observed in perpendicular direction $[2,3]$. In such case the crossfield response can cause significant error.

Theoretical response is compared to the measured results. The crossfield response was measured by two sets of the Helmholtz coils, one generating field in the sensitive axis of the tested sensor, the other perpendicular. Ferromagnetic objects which could deform the magnetic field or cause nonlinearity were removed from the vicinity of the test setup. Each characteristics was measured several times to check repeatability; the data were not averaged, but they are shown in the same plot. The perpendicularity of the crossfield coil was adjusted to give minimum response. The residual linear response was corrected in the collected dataset.

\section{AMR sensors}

Crossfield sensitivity is inherent for all AMR sensors [4]. For the compass based on the unflipped Honeywell HMC 1001 the azimuth error caused by crossfield is $1 \mathrm{deg}$.

The crossfield is in the easy direction of the sensor permalloy stripe magnetization, so AMR sensors are very sensitive to it.

The basic equation for the response of the barber-pole sensor bridge is [5]

$$
R=R_{0}^{\prime}+\Delta R \frac{H_{Y}}{H_{x}+H_{0}} \sqrt{1-\left(\frac{H_{y}}{H_{x}+H_{0}}\right)^{2}}
$$

In [4] we have used simplification for $\left|\mathrm{H}_{\mathrm{x}}\right|,\left|\mathrm{H}_{\mathrm{y}}\right|<<\left|\mathrm{H}_{0}\right|$ and we finally arrived to

$$
V_{1}=\frac{a H_{y}}{H_{x}+H_{0}}
$$

where

$\mathrm{H}_{\mathrm{x}}$ is the crossfield

$\mathrm{H}_{\mathrm{y}}$ is the measured field

$\mathrm{H}_{0}$ is the (constant) anisotropy field

a is the sensitivity constant 
The anisotropy field for Honeywell $\mathrm{HMC} 1001$ is $\mathrm{H}_{0}=0.8 \mathrm{mT}$. Increasing $\mathrm{H}_{0}$ leads to suppression of the crossfield effect, but also to the decrease of sensitivity, which is accompanied by the increase of the magnetic field sensor noise.

Many AMR sensors are stabilized by flipping, i.e. reversing the remanent magnetization of the magnetic layer by applying SET/RESET pulses into the flipping coil. Flipping pulses should have large amplitude to restore single-domain state of the sensor core [6]. The flipping field has the same direction as the crossfield. In [4] we have shown that flipping reduces sensitivity to crossfield. Later in this paper we will show that this is limited to small fields.

Mohamadabadi et al. [7] used more precise approximation of the AMR equations

$$
V_{1}=\frac{a H_{y}}{\sqrt{\left(H_{x}+H_{0}\right)^{2}+H_{y}^{2}}}
$$

and they developed and experimentally verified correction method which reduces the crossfield error without flipping by the factor of 8 , and with flipping by the factor of 9 . The problem of the mentioned correction methods is that they work only at small fields and they also require two or three sensors.

For perpendicular field larger than $200 \mu \mathrm{T}$ the AMR sensors exhibit loss of sensitivity. Fig. 1 shows the characteristics of the HMR2003 AMR module which is based on HMC1000 sensors. The flipping was switched-off and the characteristics was measured for several values of the crossfield. Applying crossfield has similar effect as increasing $\mathrm{H}_{0}$ : the sensitivity is decreased and the full-scale range is increased. This behaviour seems to be useful for increasing of the sensor range more effectively than using offset stripe, which has only $50 \mathrm{~mA} / \mathrm{G}$ field factor.

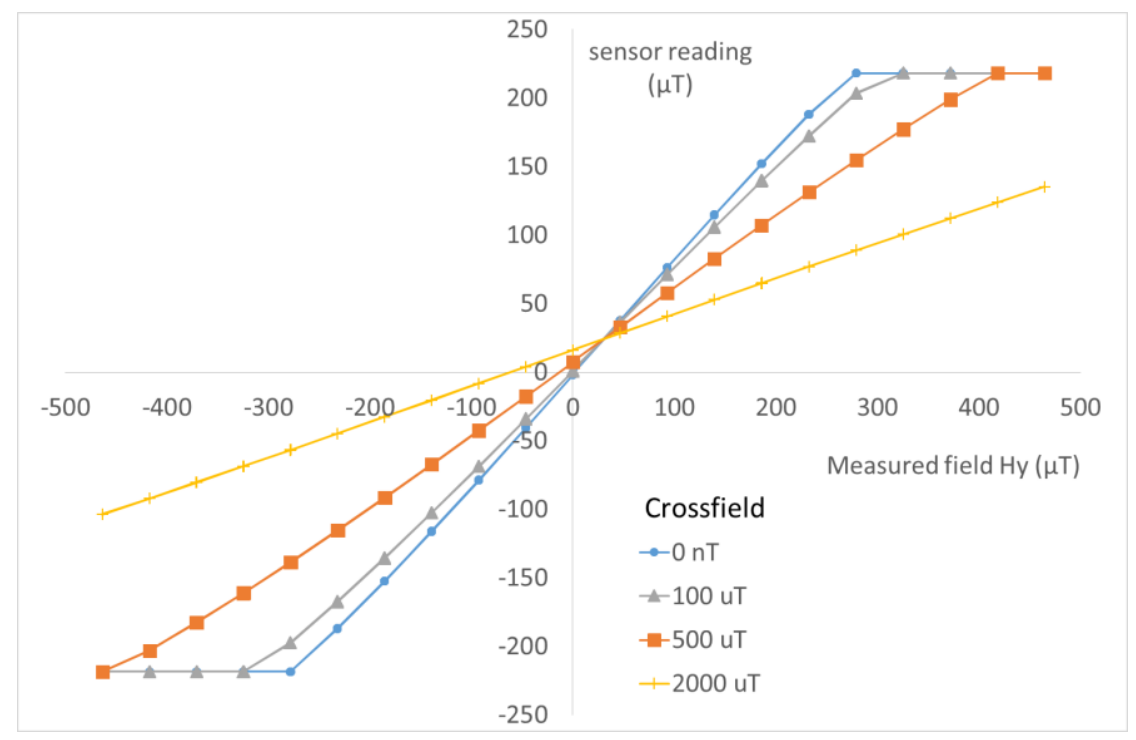

Fig. 1 AMR magnetometer characteristics for several values of the crossfield. Measured without flipping.

However, for practical situations this mode fails when the flipping is on, as shown in Fig. 2. When the flipping is applied, the sensor output polarity is periodically changing. The sensor output is therefore 
processed by synchronous detector controlled by the flipping signal. Two subsequent readings are subtracted giving double sensitivity.

Even for small crossfield the small field sensor characteristics is not affected, as the SET/RESET pulses erase the crossfield effect. The large field response is no longer reversed by flipping, which results in the sensor sensitivity falling to zero. Thus the claim that flipping increases the AMR sensors stability [8] is valid only for small field range.

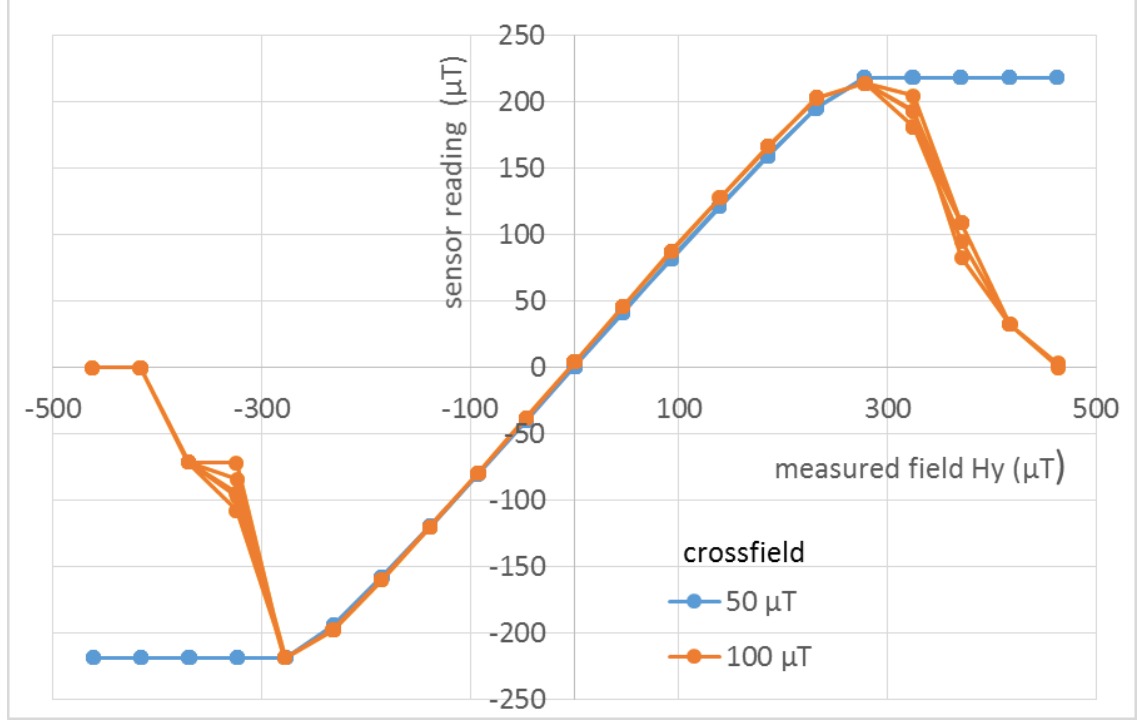

Fig. 2 Characteristics of the flipped AMR sensor without and with $50 \mu \mathrm{T}$ crossfield

The flipped sensor response is dramatically changed for larger crossfield values. This situation is illustrated by Fig. 3. The crossfield of $\mathrm{H}_{\mathrm{x}}=250 \mu \mathrm{T}$ is here so large, that flipping cannot fully reverse the core magnetization. For crossfield of $\mathrm{H}_{\mathrm{x}}=500 \mu \mathrm{T}$ the magnetisation is constant, regardless the polarity of the flipping pulse. The detector output is therefore zero.

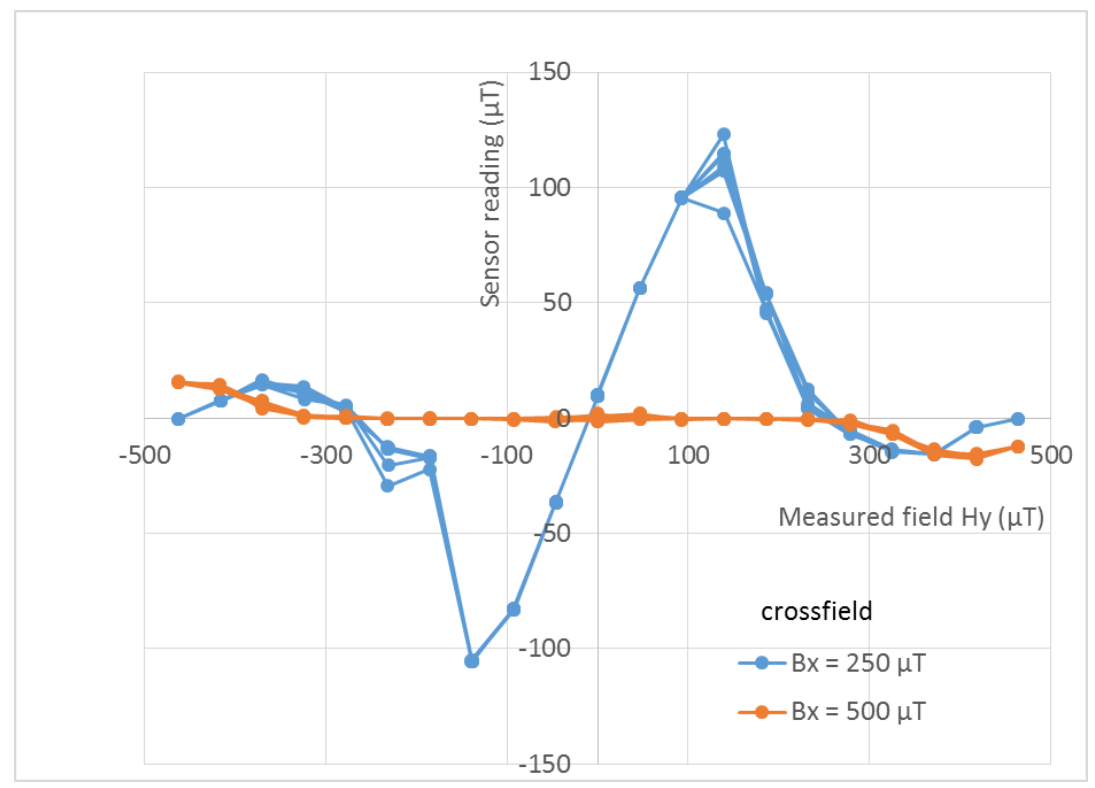

Fig. 3 Characteristics of the flipped AMR sensor with $250 \mu \mathrm{T}$ and $250 \mu \mathrm{T}$ crossfield 
If we look on the sensor output as a function of the crossfield, we finally observe the hysteresis, indicating that the sensor magnetic core is no longer in the single-domain state. Fig. 4 shows the sensor output as a function of crossfield for small values of the measured field $\mathrm{H}_{\mathrm{y}}$. For every field value in the measuring direction $\mathrm{H}_{\mathrm{y}}<200 \mu \mathrm{T}$, the response is very similar.

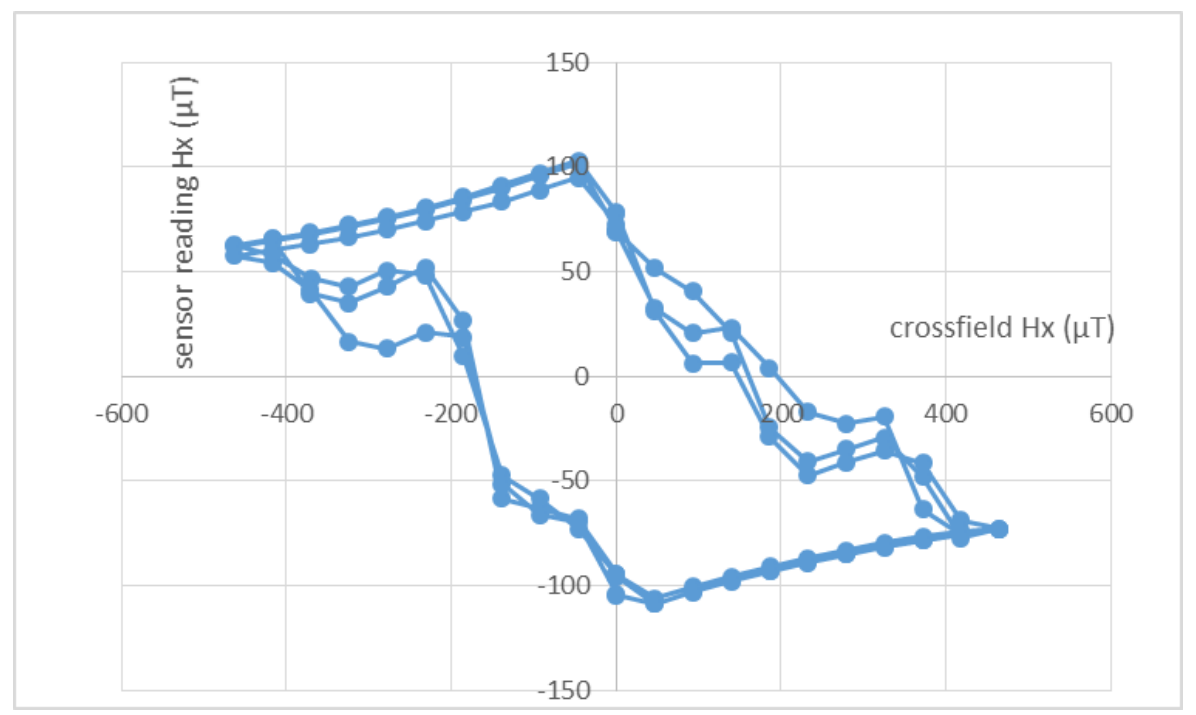

Fig. 4 Sensor output as a function of crossfield for small value of the measured field $\mathrm{H}_{\mathrm{y}}=50 \mu \mathrm{T}$ (no flipping)

For larger $\mathrm{H}_{\mathrm{y}}$ the hysteresis starts to decrease as shown in Fig. 5 and for $\mathrm{H}_{\mathrm{y}}=0.7 \mathrm{mT}$ the hysteresis disappears - the presence of the large field component the magnetization process in the strip longitudinal direction becomes rotational, this means that the hard and easy axes are now flipped. This state is interesting, but it can hardly any practical applications.

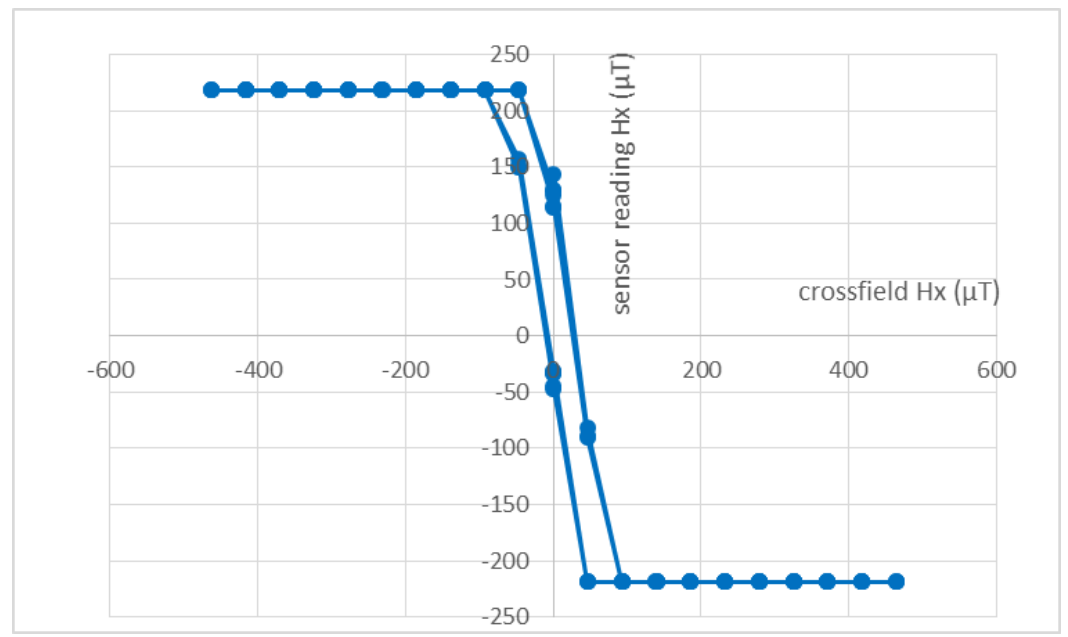

Fig. 5 AMR sensor output as a function of crossfield for very large value of the measured field $\mathrm{H}_{\mathrm{y}}=500 \mu \mathrm{T}$ (no flipping)

The same dependence for the flipped sensor is shown in Fig. 6 confirming that in this case the critical field is about $350 \mu \mathrm{T}$ for zero measured field, but only $100 \mu \mathrm{T}$ for the maximum measured field of 250 $\mu \mathrm{T}$. 


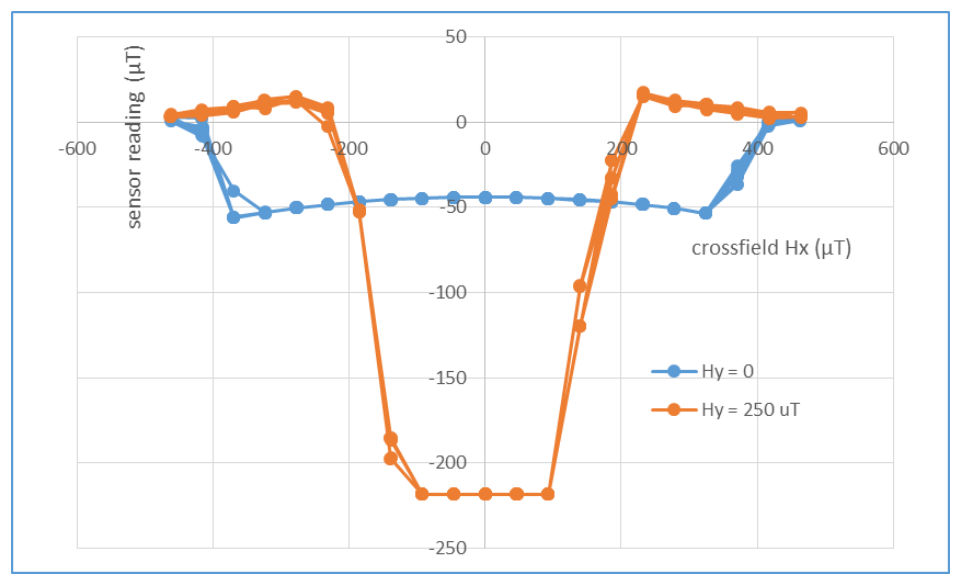

Fig. 6 Output of the flipped AMR sensor as a function of the crossfield for $\mathrm{B}_{\mathrm{y}}=0$ and $\mathrm{B}_{\mathrm{y}}=250 \mu \mathrm{T}$.

We may conclude that AMR sensors are very sensitive to crossfield larger than the critical value, which is about $350 \mu \mathrm{T}$ for the Honeywell HMC1001. It should be noted that this critical value is one order of magnitude lower than the anisotropy field $\mathrm{H}_{0}$. This value is decreasing with increased value of the measured field $\mathrm{H}_{\mathrm{y}}$.

For larger values of the crossfield the sensor characteristics are heavily distorted due to the fact that the single-domain state is broken. However, some reading is still possible for sensor with no flipping. Once the flipping is on, the sensor output in useless.

The exact value of the critical crossfield is clear from Fig. 7. The measured field $\mathrm{H}_{\mathrm{x}}$ was kept zero and the response to the crossfield was measured, while the amplitude of the crossfield was slowly increased. The figure shows that for $\mathrm{H}_{\mathrm{x}}=370 \mu \mathrm{T}$ the response in nonlinear (as expected), without any hysteresis. The onset of hysteresis signalling first closure domains appeared at $\mathrm{H}_{\mathrm{x}}=380 \mu \mathrm{T}$, which corresponds to drop of the sensitivity of the flipped sensor (Fig. 6). With increasing maximum $\mathrm{H}_{\mathrm{x}}$ the hysteresis rapidly increases.

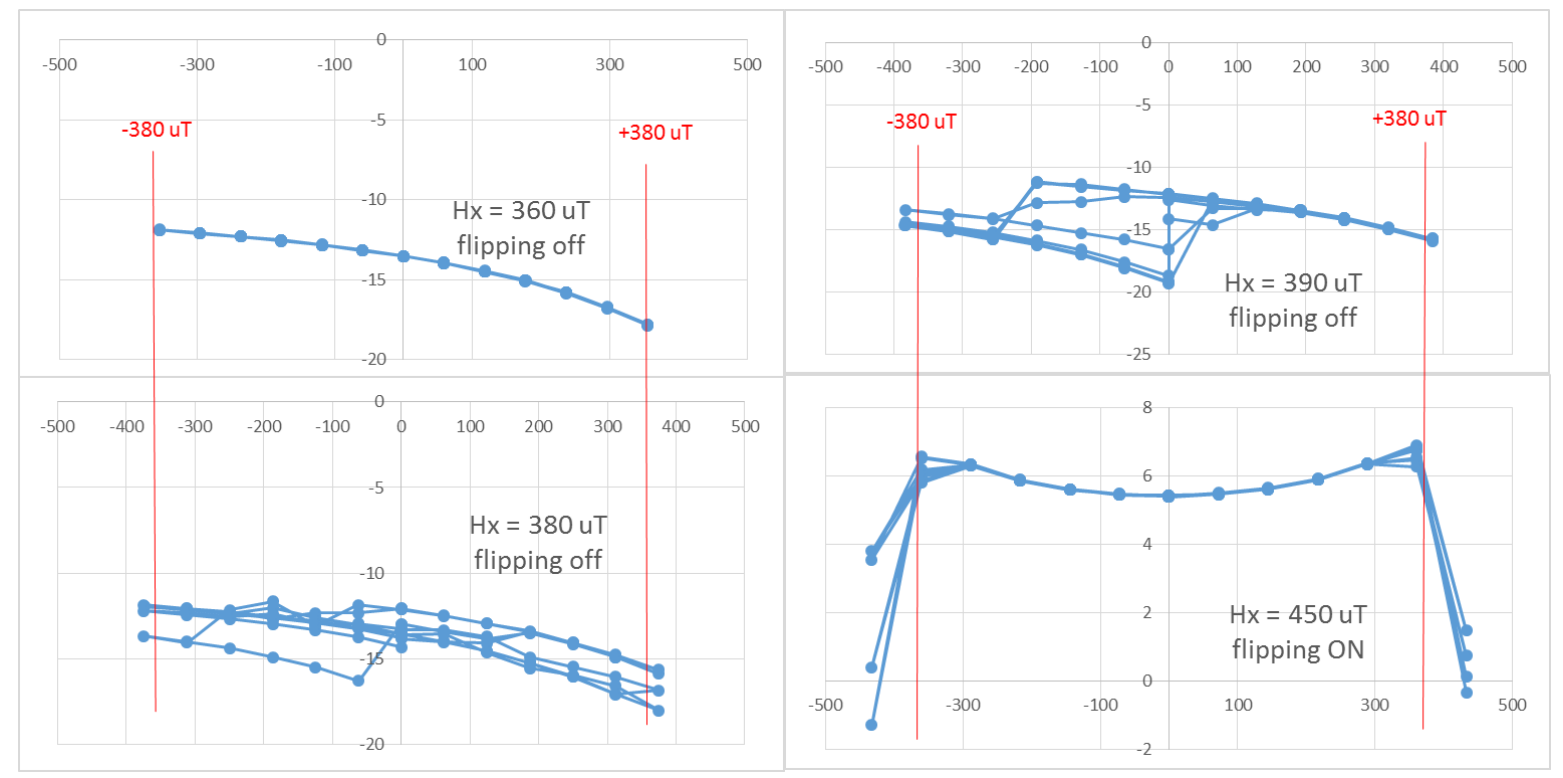

Fig. 7 Sensor response for slowly increasing value of the crossfield $\mathrm{H}_{\mathrm{x}}$. 


\section{Fluxgate sensors}

Crossfield was reported also for fluxgate sensors. First it was observed on the data from the Magsat magnetometer and verified by measurement and simulations [9].

In general, the effect of the crossfield is suppressed by high demagnetization factor in the direction perpendicular to the sensing axis [10]. Sensors with stripe or racetrack cores are therefore more resistant to the crossfield than ring-core sensors.

For the PCB fluxgate the crossfield error was $0.3 \%$. Crossfield in fluxgate is a second-order error. It is caused by core non-homogeneity and imperfections in the geometry of the core and windings [11]. However, crossfield effect was not examined in the wider field range and it was never measured on microfabricated sensors.

Microfabricated fluxgates appeared recently. They replace the CMOS-based fluxgates with flat coils, which had poor coupling between the coil and core. Lu used microfabricated solenoid coils for excitation and flat coils for pick-up. The core was formed by two stripes [12]. Rectangular closed-core fluxgate of this type having solenoid coils both for excitation and pick-up was described in [13]. Closed core has an advantage in better excitation efficiency leading to lower perming and lower power consumption, but it is more sensitive to crossfield than the rod-type core.

We made our measurements on DRV 425 integrated fluxgate manufactured by Texas Instruments [14]. This sensor has open core formed by dual permalloy stripes of about $1 \mathrm{~mm}$ length. The device model was described in [15]. Internal signal processing is analog, which is still giving better results than digital fluxgates such as described in [16].

Fig. 8 shows that the TI DRV 425 integrated fluxgate has only $0.1 \%$ crossfield error for perpendicular fields up to $2 \mathrm{mT}$. The crossfield of this magnitude has no effect on the sensor linearity error which is also $0.1 \%$ as shown in Fig. 9. For the crossfield of this magnitude AMR sensors completely fail. Even for the crossfield of $\mathrm{H}_{\mathrm{x}}=10 \mathrm{mT}$ the response of this sensor has only $0.1 \%$ hysteresis and the linearity error of 0.4 $\%$.

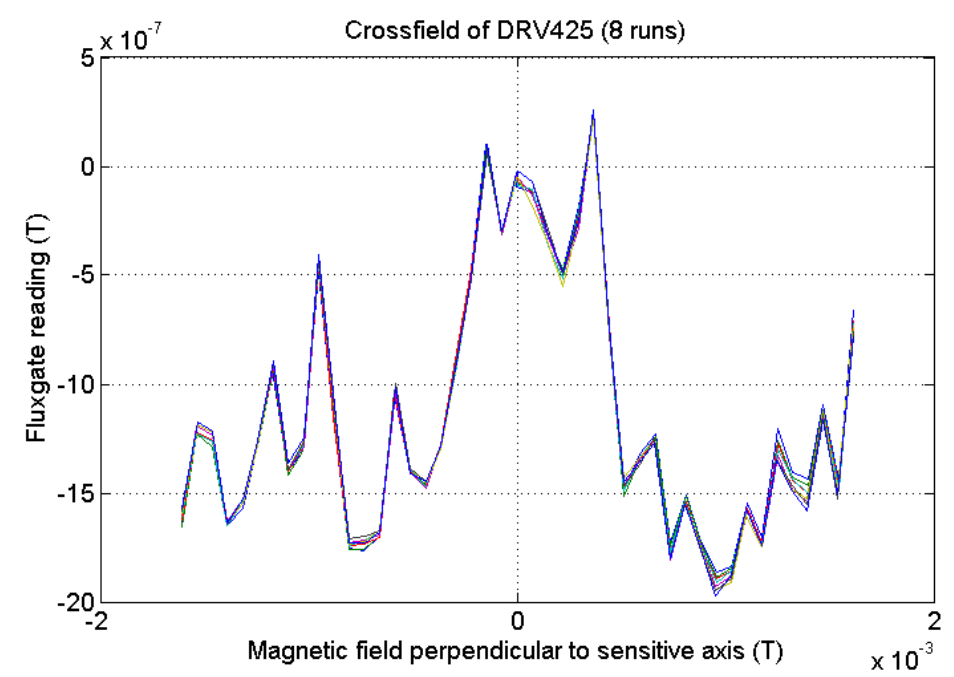

Fig. 8 - Crossfield sensitivity of the integrated fluxgate DRV 425. 


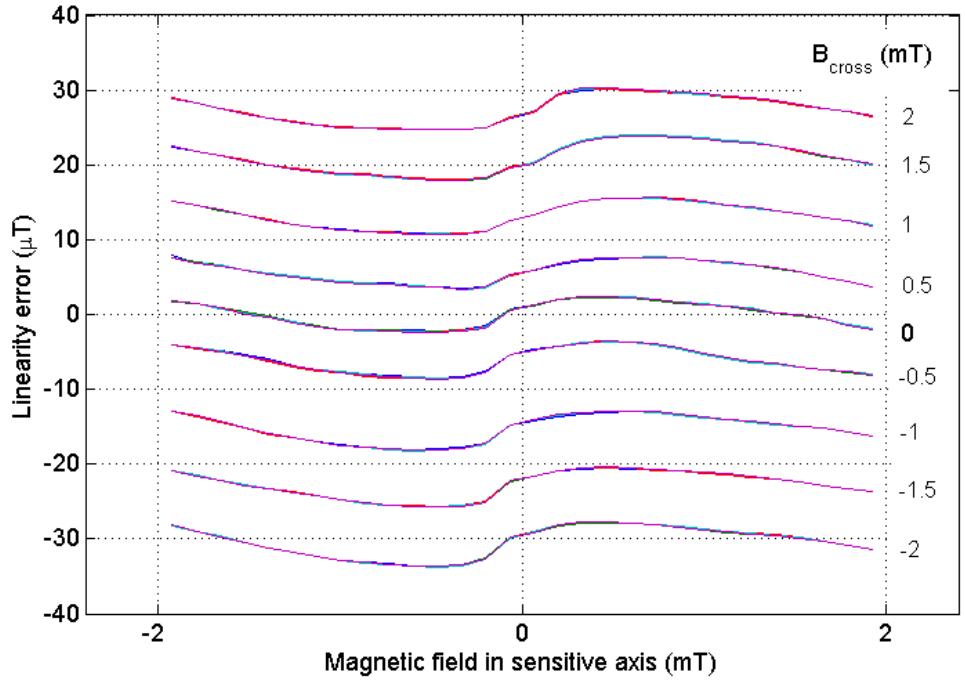

Fig.9 - Linearity error of the integrated fluxgate DRV 425. The curve parameter is the crossfield.

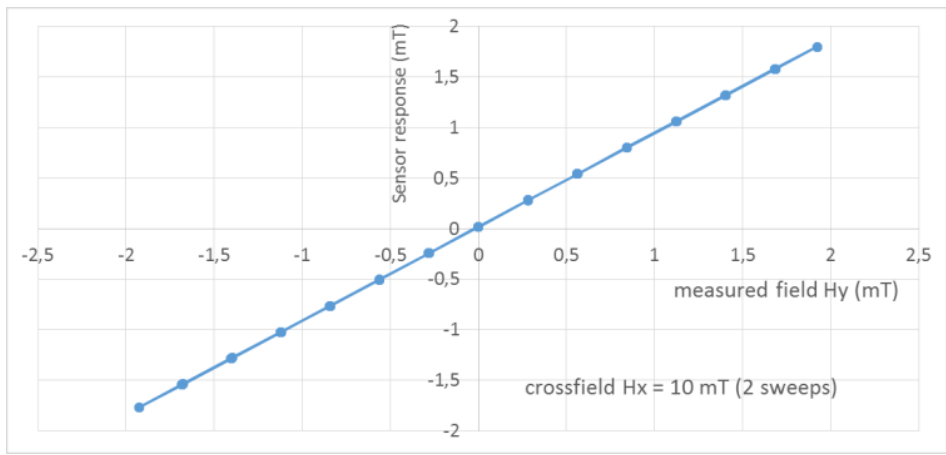

Fig. 10: DRV425 sensor response for the crossfield of $\mathrm{H}_{\mathrm{x}}=10 \mathrm{mT}$

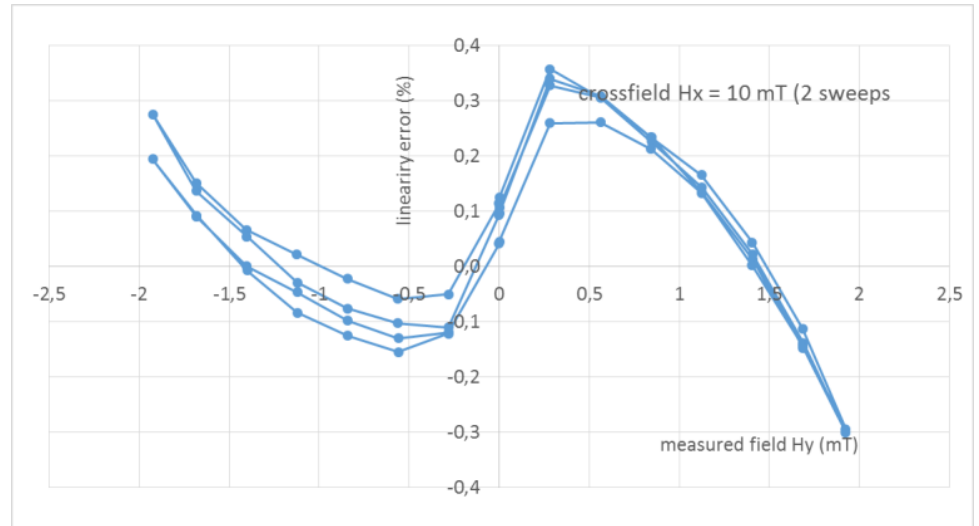

Fig. 11: DRV linearity error for the crossfield of $\mathrm{H}_{\mathrm{x}}=10 \mathrm{mT}$

\section{Conclusions}


AMR sensors are by principle sensitive to crossfield, but if the total applied field is small (below $100 \mu \mathrm{T}$ for Honeywell HMC 1001), this sensitivity can be suppressed by feedback, flipping or by calculation. For larger fields the crossfield error becomes dramatic and due to non-linearity it cannot be compensated. As a result, barber-pole AMR sensors cannot be used in applications in which fields above $250 \mu \mathrm{T}$ can be present.

Compared to that, fluxgate sensors including microfluxgates are by principle immune against the crossfield. Unlike in AMR, crossfield sensitivity in fluxgate sensors is second-order effect, which can be kept under control by proper design.

The high crossfield resistance of the integrated fluxgate is caused by

1. high homogeneity of the deposited magnetic core compared to amorphous tapes

2. geometrical accuracy of the sensor core and windings thanks to the microfabrication technology

3. high demagnetisation factor of the stripe cores in the crossfield direction.

We show that even crossfield of $10 \mathrm{mT}$ does not cause significant degradation of the sensor precision.

High crossfield resistance allows to use the microfabricated fluxgate sensor in the vicinity of permanent magnets (for position sensing) and strong electric currents (for current sensing in multiphase systems). The compass error caused by the measured crossfield error would be below $0.1 \mathrm{deg}$. Crossfield resistance is also required for applications based on eddy-currents such as non-destructive testing and distance sensing of conductive objects.

\section{ACKNOWLEDGMENT}

This work was supported by the Grant agency of the Czech Republic through the project "New methods for the measurement of electric currents" (GACR 17-19877S).

\section{References}

[1] P. Ripka, M. Janosek, M. Butta, S. W. Billingsley, E. Wakefield: Crossfield error in fluxgate and AMR sensors, Journal of Electrical Engineering. 2010, vol. 61, no. 7/s, p. 13-16.

[2] A. Fujii, T. Adachi, and Y. Gotoh, Examination of inspecting method of opposite side defect in steel plate using AC and DC magnetic field, International Journal of Applied Electromagnetics and Mechanics, vol. 52, no. 3-4, pp. 1045-1052, 2016

[3] Y. Sun, Y., Z. Ye, G. Yang, Guangyou, D. Li, S. Liu, Y. Kang, M. Gu, Ch. Liu, Pulsed electric flux leakage (PEFL) techniques for defects detection and characterization, International Journal of Applied Electromagnetics and Mechanics, vol. 52, no. 3-4, pp. 1099-1106, 2016

[4] J. Kubik, J. Vcelak, P. Ripka: On Cross-Axis Effect of the Anisotropic Magnetoresistive Sensors, Sensors \& Actuators A129 (2006), pp 15-19

[5] Tumanski: Thin Film Magnetoresistive Sensors, IoP Publ, 2001, p. 17-27RIPKA, P. -

[6] P. Ripka, M. Janosek, M. Butta: Crossfield Sensitivity in AMR Sensors, IEEE Trans. Magn., 45 (2009), 4514 - 4517

[7] H. Hauser, P.L. Fulmek, P. Haumer, M. Vopalensky, P. Ripka, Flipping field and stability in anisotropic magnetoresistive senzors, Sensors \& Actuators A 106 (2003), 34-37, pp 121-125

[8] Mohamadabadi, K.; Coillot, Ch.; Hillion, M.: New Compensation Method for Cross-Axis Effect for Three-Axis AMR Sensors, IEEE Sens. J. 13 (2013), pp. 1355-1362

[9] Brauer, P. et al: Transverse effect in fluxgate sensors, Sens. Actuators A 59 (1997), 70-74

[10] P.Ripka, W. Billingsley: Crossfield effect at fluxgate, Sensors and Actuators A 81 (2000), 176-179.

[11] M. Janosek, M. Butta, P. Ripka: Two sources of cross-field error in racetrack fluxgate, J. App. Phys., 107, 09E713 (2010).

[12] Lu Chih-Cheng, Wen-Sheng Huang, Yu-Ting Liu, Jen-Tzong Jeng: Design, Fabrication, and Characterization of a 3-D CMOS Fluxgate Magnetometer, IEEE Trans. Magn. 47 (2011),3752-3756.

[13] Lei Chong, Yan Liu, Xue-Cheng Sun, Tao Wang, Zhen Yang, and Yong Zhou: Improved Performance of Integrated Solenoid Fluxgate Sensor Chip Using a Bilayer Co-Based Ribbon Core, IEEE Sens. J. 15 (2015), 5010-5015.

[14] M. F. Snoeij; V. Schaffer; S. Udayashankar; M. V. Ivanov, "Integrated Fluxgate Magnetometer for Use in Isolated Current Sensing," in IEEE Journal of Solid-State Circuits , vol.PP, no.99, pp.1-11, doi: 10.1109/JSSC.2016.2554147

[15] Gaskill Steven G.; Weisshaar Andreas: Compact Equivalent Circuit Modeling of Microfluxgate Devices With Thin-Film Magnetic Cores, IEEE Trans. Magn. 52(2016), Article \#: 4002808

[16] M. Kashmiri et al., "A $200 \mathrm{kS} / \mathrm{s}$ integrated-fluxgate differential magnetic-to-digital converter with an oversampling compensation loop for contactless current sensing," in IEEE ISSCC Dig. Tech. Papers, 2015, pp. 490-491. 
